\title{
IUFOST2006/591 Pulsed Electric Fields (PEF) Processing of Meat
}

\author{
S. Toepfl, V. Heinz and D. Knorr \\ Berlin University of Technology, Department Food Biotechnology and Food Process Engineering, \\ Koenigin-Luise-Str. 22, 14195 Berlin, Germany \\ stefan.toepfl@tu-berlin.de
}

\begin{abstract}
A PEF treatment can be utilized to enhance processes where mass transport is required, such as drying, marination or curing of meat products. Salt (often in combination with phosphate or nitrite) is applied for preservation; spices are added to develop taste and aroma profiles. In case of raw ham (Parma- or Serrano type) a long-term curing and air drying is applied. It was shown that the fermentation of raw sausages (Salami type) can be accelerated by improving the availability of intracellular liquid. A PEF treatment of whole pig haunches during production of Serrano type ham revealed an increased uptake of salt after hand salting. The impact of a PEF treatment at a field strength from 1 to $3 \mathrm{kV} / \mathrm{cm}$ on drying of pork meat was investigated. For production of cooked ham pickling brine was injected, the impact of a PEF treatment, the addition of phosphate and application of a tumbling step on water binding capacity was investigated. The impact of electric field strength, pulse number and addition of water binding agents such as phosphate was investigated. It has been shown that after a PEF treatment at $2 \mathrm{kV} / \mathrm{cm}$ and 100 pulses the brine distribution within the tissue of pork meat can be improved using a commercially available injector. Water binding capacity has been improved, resulting in a reduction of weight loss during cooking as well as softer structure of final product. REM micrographs revealed an impact of PEF-improved microdiffusion on meat tissue structure. At present an industrial scale prototype is on its way to realisation.
\end{abstract}

